PROCEEDINGS OF THE

AMERICAN MATHEMATICAL SOCIETY

Volume 134, Number 9, September 2006, Pages 2571-2576

S 0002-9939(06)08259-1

Article electronically published on February 17, 2006

\title{
ON OPERATORS WHICH COMMUTE WITH ANALYTIC TOEPLITZ OPERATORS MODULO THE FINITE RANK OPERATORS
}

\author{
KUNYU GUO AND KAI WANG
}

(Communicated by Joseph A. Ball)

\begin{abstract}
It is shown that an operator $S$ on the Hardy space $H^{2}\left(\mathbb{D}^{n}\right)$ (or $H^{2}\left(\mathbb{B}_{n}\right)$ ) commutes with all analytic Toeplitz operators modulo the finite rank operators if and only if $S=T_{g}+F$. Here $F$ is a finite rank operator, and in the case $n=1, g$ is a sum of a rational function and a bounded analytic function, and in the case $n \geq 2, g$ is a bounded analytic function.
\end{abstract}

\section{INTRODUCTION}

Davidson [Da] studied when an operator $S$ on the classical Hardy space $H^{2}(\mathbb{D})$ essentially commutes with all analytic Toeplitz operators. He proved that commutators $\left[S, T_{f}\right]=S T_{f}-T_{f} S$ are compact for all $f \in H^{\infty}(\mathbb{D})$ if and only if $S=T_{g}+K$, where $g \in H^{\infty}(\mathbb{D})+C(\mathbb{T})$ and $K$ is compact. Guo Guo generalized this result to the Hardy space over the unit ball $\mathbb{B}_{n}$.

A natural problem is to characterize operators $S$ such that the commutators $\left[S, T_{f}\right]$ belong to the Schatten-von Neumann class $\mathcal{L}^{p}$ for all $f \in H^{\infty}$. Note the inclusion

$$
\mathcal{L}^{0} \subset \cdots \subset \mathcal{L}^{1} \subset \cdots \subset \mathcal{L}^{\infty}
$$

where $\mathcal{L}^{0}$ is the class of all finite rank operators, and $\mathcal{L}^{\infty}$ is the class of all compact operators. Therefore, Davidson's work completed the case of $\mathcal{L}^{\infty}$ of the classical Hardy space in the above sequence. Furthermore, on the classical Hardy space $H^{2}(\mathbb{D}), \mathrm{Gu}$ Gu1] showed that for each $f \in H^{\infty}(\mathbb{D}),\left[S, T_{f}\right]=S T_{f}-T_{f} S \in \mathcal{L}^{0}$ if and only if $S=T_{g}+F$, where $g$ is a sum of a bounded analytic function and a rational function, and $F$ is a finite rank operator. However, the proof in Gu1 is considerably technical because $\mathrm{Gu}$ solved a more general problem from which the above-mentioned result is a consequence. In this note, we will generalize Gu's result to the case of higher dimension, and in the dimension $n=1$, our proof is different from the proof in Gu1. The following is the main result in this note.

Theorem 1.1. Let $S$ be a bounded linear operator on $H^{2}\left(\mathbb{D}^{n}\right)$ (or $\left.H^{2}\left(\mathbb{B}_{n}\right)\right)$. Then $\left[S, T_{f}\right]$ is of finite rank for all $f \in H^{\infty}$ if and only if $S=T_{g}+F$. Here $F$ is a finite rank operator, and in the case $n=1, g$ is a sum of a rational function and a bounded analytic function, and in the case $n \geq 2, g$ is a bounded analytic function.

Received by the editors December 13, 2004 and, in revised form, March 18, 2005.

2000 Mathematics Subject Classification. Primary 47B35, 47B20.

Key words and phrases. Hardy space, Toeplitz operator, finite rank operator.

(C)2006 American Mathematical Society 


\section{Preliminaries}

Let $\mathbb{D}$ be the open unit disk of the complex plane and $\mathbb{T}$ the unit circle. For $n \geq 1$, let $\mathbb{D}^{n}$ and $\mathbb{T}^{n}$ be the unit polydisk and $n$-torus, respectively. The Hardy space $H^{2}\left(\mathbb{D}^{n}\right)$ is the closure of all polynomials in $L^{2}\left(\mathbb{T}^{n}\right)$ (with respect to the measure $d \theta_{1} \cdots d \theta_{n} /(2 \pi)^{n}$ on $\left.\mathbb{T}^{n}\right)$. Let $P$ be the orthogonal projection from $L^{2}\left(\mathbb{T}^{n}\right)$ onto $H^{2}\left(\mathbb{D}^{n}\right)$. The Toeplitz operator $T_{f}: H^{2}\left(\mathbb{D}^{n}\right) \rightarrow H^{2}\left(\mathbb{D}^{n}\right)$ with symbol $f \in L^{\infty}\left(\mathbb{T}^{n}\right)$ is defined by $T_{f}(h)=P(f h)$ for all $h \in H^{2}\left(\mathbb{D}^{n}\right)$. The Hankel operator $H_{f}$ with symbol $f$ is defined as $H_{f} h=(I-P)(f h)$ for all $h \in H^{2}\left(\mathbb{D}^{n}\right)$. For $f, g \in L^{\infty}\left(\mathbb{T}^{n}\right)$, Toeplitz and Hankel operators are connected by the following formula:

$$
T_{f g}-T_{f} T_{g}=H_{\bar{f}}^{*} H_{g}
$$

Let us recall the Hardy space $H^{2}\left(\mathbb{B}_{n}\right)$ over the unit ball. Let $\mathbb{B}_{n}$ be the open unit ball in $\mathbb{C}^{n}$, and $\partial \mathbb{B}_{n}$ its boundary. The Hardy space $H^{2}\left(\mathbb{B}_{n}\right)$ is the closure of all polynomials in $L^{2}\left(\partial \mathbb{B}_{n}\right)$ (with respect to the unique rotation-invariant probability measure $d \sigma$ on $\left.\partial \mathbb{B}_{n}\right)$. In the same way, one can define Toeplitz and Hankel operators on the Hardy space $H^{2}\left(\mathbb{B}_{n}\right)$.

It is well known that there exist a lot of inner functions both in the unit ball $\mathbb{B}^{n}$ and the unit polydisk $\mathbb{D}^{n}[\mathrm{R} 1, \mathrm{R} 2]$. Inner functions will play an important role in this note. In what follows we will use $H^{2}$ to denote the Hardy space $H^{2}\left(\mathbb{B}_{n}\right)$ or $H^{2}\left(\mathbb{D}_{n}\right)$.

Lemma 2.1. Let $\eta$ be a nonconstant inner function. Then $T_{\bar{\eta}^{n}} \rightarrow 0(S O T)$ on the Hardy space $H^{2}$. Furthermore, for each compact operator $K$, we have $T_{\bar{\eta}^{n}} K \stackrel{\|\cdot\|}{\rightarrow} 0$ as $n \rightarrow \infty$.

Proof. Let $K_{\lambda}$ be the reproducing kernel of $H^{2}$. Take $f=\sum a_{i} K_{\lambda_{i}}$ to be a finite linear combination of reproducing kernels. Note that

$$
T_{\bar{\eta}^{n}}\left(\sum a_{i} K_{\lambda_{i}}\right)=\sum a_{i}{\overline{\eta\left(\lambda_{i}\right)}}^{n} K_{\lambda_{i}} .
$$

Hence

$$
\left\|T_{\bar{\eta}^{n}}\left(\sum a_{i} K_{\lambda_{i}}\right)\right\| \leq \sum\left|a_{i}\right|\left|\eta\left(\lambda_{i}\right)\right|^{n}\left\|K_{\lambda_{i}}\right\| \rightarrow 0
$$

as $n \rightarrow \infty$. Because the above linear combinations are dense in $H^{2}, T_{\bar{\eta}^{n}} \rightarrow 0$ $(S O T)$. For a rank one operator $f \otimes g$ (here $f \otimes g(h)=\langle h, g\rangle f)$, we have

$$
T_{\bar{\eta}^{n}} f \otimes g=\left(T_{\bar{\eta}^{n}} f\right) \otimes g .
$$

Note that the set of all finite rank operators is dense in all compact operators, the desired result follows.

The following lemma may be known by many people. We note it here.

Lemma 2.2. Let $\left\{T_{\alpha}\right\}$ be a net of operators on $H^{2}$ such that $T_{\alpha} \rightarrow T$ (WOT). If there exists a natural number $M$ such that $\operatorname{rank} T_{\alpha} \leq M$, then

$$
\operatorname{rank} T \leq M \text {. }
$$

Proof. If $\operatorname{rank} T \geq M+1$, then there exist $\left\{f_{i}\right\}_{i=1}^{M+1}$ and $\left\{g_{i}\right\}_{i=1}^{M+1}$ such that

$$
\operatorname{det}\left[\left\langle T f_{i}, g_{j}\right\rangle\right]_{1 \leqslant i, j \leqslant M+1} \neq 0 \text {. }
$$

Set

$$
d_{\alpha}=\left[\left\langle T_{\alpha} f_{i}, g_{j}\right\rangle\right]_{1 \leqslant i, j \leqslant M+1}
$$


Then $d_{\alpha}=0$ since $\operatorname{rank} T_{\alpha} \leq M$. This leads to a contradiction since

$$
d_{\alpha} \rightarrow \operatorname{det}\left[\left\langle T f_{i}, g_{j}\right\rangle\right]_{1 \leqslant i, j \leqslant M+1} \neq 0 .
$$

Lemma 2.3. Let $S$ be an operator on $H^{2}$. If $\left[S, T_{f}\right]$ is of finite rank for all $f \in H^{\infty}$, then there exists a natural number $M$ such that

$$
\operatorname{rank}\left[S, T_{f}\right] \leq M \text {. }
$$

Proof. Let $\Gamma_{n}=\left\{f: \operatorname{rank}\left[S, T_{f}\right] \leq n\right\}$. By Lemma 2.2, $\Gamma_{n}$ is a norm closed subset of $H^{\infty}$. Since $H^{\infty}$ is a Banach space and $H^{\infty}=\bigcup_{n} \Gamma_{n}$, the Baire Category Theorem implies that there exists a natural number $N$ such that $\Gamma_{N}$ contains an open subset of $H^{\infty}$. This means that the set $\left\{f-g: f, g \in \Gamma_{N}\right\}$ is a neighborhood of the function $f=0$. Thus for each $h \in H^{\infty}$, there exists a real number $\gamma$ and two functions $f, g \in \Gamma_{N}$ such that $h=\gamma(f-g)$ and hence

$$
\operatorname{rank}\left[S, T_{h}\right] \leq \operatorname{rank}\left[S, T_{f}\right]+\operatorname{rank}\left[S, T_{g}\right] \leqq 2 N .
$$

The next proposition says that Toeplitz operators on the Hardy space $H^{2}$ can be completely characterized by algebraic equations.

Proposition 2.1. For a bounded linear operator $T$ on $H^{2}$, then $T$ is a Toeplitz operator if and only if $T_{\eta}^{*} T T_{\eta}=T$ for each inner function $\eta$.

Proof. We will prove the proposition in the case of the unit ball, and the same reasoning is valid in the unit polydisk. Set

$$
\mathcal{A}=\left\{\bar{\eta} h: \eta \text { are inner functions, } h \in H^{2}\right\} .
$$

Then $\mathcal{A}$ is a dense linear subspace of $L^{2}\left(\partial \mathbb{B}_{n}\right)$ by [R1, Theorem 11.4]. Define a map

$$
\Phi: \mathcal{A} \rightarrow \mathbb{C}
$$

by $\Phi(\bar{\eta} h)=\langle h, T \eta\rangle$. Then $\Phi$ is well defined and linear. In fact, if $\bar{\eta}_{1} h_{1}=\bar{\eta}_{2} h_{2}$, then we have

$$
\begin{aligned}
\Phi\left(\bar{\eta}_{1} h_{1}\right) & =\left\langle h_{1}, T \eta_{1}\right\rangle=\left\langle h_{1}, T_{\eta_{2}}^{*} T T_{\eta_{2}} \eta_{1}\right\rangle \\
& =\left\langle\eta_{2} h_{1}, T \eta_{2} \eta_{1}\right\rangle=\left\langle\eta_{1} h_{2}, T \eta_{1} \eta_{2}\right\rangle \\
& =\left\langle h_{2}, T_{\eta_{1}}^{*} T T_{\eta_{1}} \eta_{2}\right\rangle=\left\langle h_{2}, T \eta_{2}\right\rangle \\
& =\Phi\left(\bar{\eta}_{2} h_{2}\right) .
\end{aligned}
$$

So, $\Phi$ is well defined. The same reasoning shows that $\Phi$ is linear. From the definition of $\Phi$,

$$
|\Phi(\bar{\eta} h)| \leq\|T\|\|h\|=\|T\|\|\bar{\eta} h\| .
$$

So, $\Phi$ is a bounded linear functional on $\mathcal{A}$. Since $\mathcal{A}$ is dense in $L^{2}\left(\partial \mathbb{B}_{n}\right)$, there exists a unique $\phi \in L^{2}\left(\partial \mathbb{B}_{n}\right)$ such that

$$
\Phi(\bar{\eta} h)=\langle\bar{\eta} h, \phi\rangle .
$$

Since $\mathcal{A}$ is dense in $L^{2}\left(\partial \mathbb{B}_{n}\right)$ and

$$
|\langle\bar{\eta} h, \phi\rangle|=\left|\int_{\partial \mathbb{B}_{n}}(\bar{\eta} h) \bar{\phi} d \sigma\right| \leq\|T\|\|\bar{\eta} h\|,
$$

we see $\phi \in L^{\infty}$. From the equalities

$$
\langle h, T \eta\rangle=\Phi(\bar{\eta} h)=\langle\bar{\eta} h, \phi\rangle=\langle h, \phi \eta\rangle=\left\langle h, T_{\phi} \eta\right\rangle,
$$


and note that the set of all finite linear combinations of inner functions is dense in $H^{2}$ [R1, Theorem 11.1], we obtain $T=T_{\phi}$.

By Kronecker's result about a finite rank Hankel operator, $H_{g}$ on $H^{2}(\mathbb{D})$ is of finite rank if and only if $g$ is the sum of a rational function and a bounded analytic function over the disk $\mathbb{D}\left[\mathbf{P}\right.$. For $n \geq 2$, if a Hankel operator $H_{g}$ on $H^{2}\left(\mathbb{D}^{n}\right)$ has finite rank, then it must be zero (see Gu2, GuZ]). In the case of unit ball, the following proposition may be known by many people.

Proposition 2.2. Assume $n \geq 2$ and a Hankel operator $H_{g}$ is of finite rank. Then $H_{g}=0$, that is, $g \in H^{\infty}$.

Proof. Setting $M=\operatorname{ker} H_{g}$, then $M$ is a finitely codimensional multiplier invariant subspace. As a finitely codimensional multiplier invariant subspace, $M$ is generated by finitely many polynomials $P_{1}, \ldots, P_{m}$, and the set $\bigcap_{i=1}^{m} Z\left(P_{i}\right)$ is finite CG, Corollary 2.2.6], where $Z(P)$ denotes the set of zero points of a polynomial $P$. From $H_{g} P_{i}=0$, we have $Q_{i}=g P_{i} \in H^{\infty}, \quad i=1, \ldots, m$. On $\partial \mathbb{B}_{n}$, noting

this implies

$$
g=\frac{Q_{1}}{P_{1}}=\cdots=\frac{Q_{m}}{P_{m}}
$$

$$
\frac{Q_{1}(z)}{P_{1}(z)}=\cdots=\frac{Q_{m}(z)}{P_{m}(z)}, \quad z \in \mathbb{B}_{n} \backslash \bigcup_{i=1}^{m} Z\left(P_{i}\right) .
$$

Putting $g(z)=\frac{Q_{1}(z)}{P_{1}(z)}$, then from the above equalities, $g$ can be analytically extended to $\mathbb{B}_{n} \backslash \bigcap_{i=1}^{m} Z\left(P_{i}\right)$. Since the set $\bigcap_{i=1}^{m} Z\left(P_{i}\right)$ is finite, $g$ has an extension on $\mathbb{B}_{n}$ by $\left[\mathrm{Kr}\right.$. Furthermore, we have $g \in H^{\infty}$. The reasoning is as follows: since the set $\bigcap_{i=1}^{m} Z\left(P_{i}\right)$ is finite, there exist $0<r<1$ and $\epsilon>0$ such that $\sum_{i=1}^{m}\left|P_{i}(z)\right|^{2}>\epsilon$ if $|z|>r$. By

$$
|g(z)|^{2}=\frac{\left|Q_{1}(z)\right|^{2}}{\left|P_{1}(z)\right|^{2}}=\ldots=\frac{\left|Q_{m}(z)\right|^{2}}{\left|P_{m}(z)\right|^{2}}=\frac{\sum_{i=1}^{m}\left|Q_{i}(z)\right|^{2}}{\sum_{i=1}^{m}\left|P_{i}(z)\right|^{2}},
$$

we see that $|g(z)|$ is bounded on $r<|z|<1$. This implies $g \in H^{\infty}$.

\section{THE PROOF OF THE MAIN THEOREM}

Before proving the main theorem we need the following lemma.

Lemma 3.1. On the Hardy space $H^{2}$, if $\left[T_{g}, T_{f}\right]$ is of finite rank for all $f \in H^{\infty}$, then in the case $n=1, g$ is a sum of a rational function and a bounded analytic function, and in the case $n \geq 2, g$ is a bounded analytic function.

Proof. By Lemma 2.3 there exists a natural number $M$ such that

$$
\operatorname{rank}\left[T_{g}, T_{f}\right] \leq M
$$

for all $f \in H^{\infty}$. Below we will give the proof of Lemma 3.1 in the case of unit ball, and the same reasoning is valid in the case of the unit polydisk. For any $h, f \in H^{\infty}$,

$$
\operatorname{rank}\left(T_{\bar{h} f g}-T_{\bar{h} f} T_{g}\right)=\operatorname{rank}\left(T_{\bar{h}}\left(T_{g} T_{f}-T_{f} T_{g}\right)\right) \leq \operatorname{rank}\left(T_{g} T_{f}-T_{f} T_{g}\right) \leq M .
$$

Note that $\left\{\bar{\eta} f: \eta\right.$ are inner functions, $\left.f \in H^{\infty}\right\}$ is $W^{*}$ dense in $L^{\infty}\left(\partial \mathbb{B}_{n}\right)$ R1, Theorem 11.2]. By Lemma 2.2, $T_{\phi g}-T_{\phi} T_{g}$ is of finite rank for all $\phi \in L^{\infty}\left(\partial \mathbb{B}_{n}\right)$. It follows that $T_{\bar{g} g}-T_{\bar{g}} T_{g}=H_{g}^{*} H_{g}$ is of finite rank, that is, $H_{g}$ is of finite rank. In the case $n=1$, by Kronecker's result about a finite rank Hankel operator, $H_{g}$ on 
$H^{2}(\mathbb{D})$ is of finite rank if and only if $g$ is a sum of a rational function and a bounded analytic function over the disk $\mathbb{D}[\mathrm{P}]$. In the case $n \geq 2$, applying Proposition 2.2 gives $g \in H^{\infty}$.

Theorem 3.1. Given a bounded linear operator $S$ on $H^{2}$, then $\left[S, T_{f}\right]$ is of finite rank for all $f \in H^{\infty}$ if and only if $S=T_{g}+F$. Here $F$ is a finite rank operator, and in the case $n=1, g$ is a sum of a rational function and a bounded analytic function, and in the case $n \geq 2, g$ is a bounded analytic function.

Remark. In the case $n=1, \mathrm{Gu}$ obtained the above result Gu1. However, the present proof is different from Gu's proof.

Proof. First we claim that $S$ has the form $S=T_{g}+F$, where $g \in L^{\infty}$, and $F$ is a finite rank operator. For this claim, pick a nonconstant inner function $\eta$, and consider the sequence $\left\{T_{\eta^{n}}^{*} S T_{\eta^{n}}\right\}$. Note that $\left\{T_{\eta^{n}}^{*} S T_{\eta^{n}}\right\}$ is a bounded set, and hence without a loss of generality we may assume that $\left\{T_{\eta^{n}}^{*} S T_{\eta^{n}}\right\}$ converges to a operator $A$ in the weak operator topology (if not, we can choose a subnet). It follows that

$$
S-A=(\mathrm{WOT}) \lim \left(S-T_{\eta^{n}}^{*} S T_{\eta^{n}}\right) .
$$

Applying Lemma 2.3 shows that there exists a natural number $M$ such that $\operatorname{rank}\left[S, T_{f}\right] \leq M$ for all $f \in H^{\infty}$ and hence

$$
\operatorname{rank}\left(S-T_{\eta^{n}}^{*} S T_{\eta^{n}}\right)=\operatorname{rank} T_{\eta^{n}}^{*}\left(T_{\eta^{n}} S-S T_{\eta^{n}}\right) \leq M .
$$

By Lemma 2.2, $\operatorname{rank}(S-A) \leq M$, that is, $F=S-A$ is a finite rank operator. Now we prove that $A$ is a Toeplitz operator. For each inner function $\zeta$

$$
\begin{aligned}
T_{\zeta}^{*} A T_{\zeta} & =(\mathrm{WOT}) \lim T_{\zeta}^{*} T_{\eta^{n}}^{*} S T_{\eta^{n}} T_{\zeta}=(\mathrm{WOT}) \lim T_{\eta^{n}}^{*} T_{\zeta}^{*} S T_{\zeta} T_{\eta^{n}} \\
& =(\mathrm{WOT}) \lim T_{\eta^{n}}^{*} T_{\zeta}^{*} T_{\zeta} S T_{\eta^{n}}+(\mathrm{WOT}) \lim T_{\zeta}^{*} T_{\eta^{n}}^{*}\left(S T_{\zeta}-T_{\zeta} S\right) T_{\eta^{n}} .
\end{aligned}
$$

Since $S T_{\zeta}-T_{\zeta} S$ is of finite rank, the latter is zero by Lemma 2.1. It follows that

$$
T_{\zeta}^{*} A T_{\zeta}=(\mathrm{WOT}) \lim T_{\eta^{n}}^{*} T_{\zeta}^{*} T_{\zeta} S T_{\eta^{n}}=A .
$$

Proposition 2.1 says that $A$ is a Toeplitz operator, that is, there is a $g \in L^{\infty}$ such that $A=T_{g}$. The claim follows. Using Lemma 3.1, we see that in the case $n=1$, $g$ is a sum of a rational function and a bounded analytic function, and in the case $n \geq 2, g$ is a bounded analytic function. The opposite direction is easily proved.

\section{ACKNOWLEDGMENTS}

The authors are grateful to Professor D. Zheng for his helpful suggestions. This work was partially supported by NNSFC and the Teaching and Research Award Program for Outstanding Young Teachers in Higher Education Institutions of MOE, China.

\section{REFERENCES}

[CG] X. Chen and K. Guo, Analytic Hilbert Modules, Chapman \& Hall/CRC Reserarch Notes in Mathematics, 433, 2003. MR1988884(2004d:47024)

[Da] K. Davidson, On operator commuting with Toeplitz operators modulo the compact operators, J. Funct. Anal. 24(1977), 291-302. MR0454715 (56:12963)

[Gu1] C. Gu, On operators commuting with Toeplitz operators modulo the finite rank operators, J. Funct. Anal. 215(2004), 178-205. MR2085114 (2005c:47033)

[Gu2] C. Gu, Some algebraic properties of Toeplitz and Hankel operators on polydisk, Arch. Math. 80(2003), 393-405. MR.1982839 (2004e:47039) 
[GuZ] C. Gu and D. Zheng, The semi-commutator of Toeplitz operators on the bidisk, J. Operator Theory, 38(1997), 173-193. MR1462020 (98g:47022)

[Guo] K. Guo, Essential commutants of analytic Toeplitz algebra and some related problems, Acta Math Sinica, 39(3)(1996), 300-313 [In Chinese]. MR1413350 (97i:47043)

$[\mathrm{Kr}]$ S. Krantz, Function theory of several complex variables, John Wiley \& Sons, New York, 1982. MR0635928 (84c:32001)

[R1] W. Rudin, New construction of functions holomorphic in the unit ball of $C^{n}$, Conference Board of the Mathematical Science Regional Conference Series in Mathematics, 63(1986). MR0840468 (87f:32013)

[R2] W. Rudin, Function theory in polydisks, New York, 1969.

[R3] W. Rudin, Function theory in the unit ball of $C^{n}$, Springer-Verlag, 1980. MR0601594 (82i:32002)

[P] S. Power, Hankel operators on Hilbert space, Pitman Research Notes in Math., 64, 1982. MR0666699 (84e:47037)

School of Mathematics, Fudan University, Shanghai, 200433, People's Republic of CHINA

E-mail address: kyguo@fudan.edu.cn

School of Mathematics, Fudan University, Shanghai, 200433, People's Republic of CHINA

E-mail address: 031018009@fudan.edu.cn 